\title{
Epidemic processes with immunization
}

\author{
Andrea Jiménez-Dalmaroni 1 , * and Haye Hinrichsen ${ }^{2}$ \\ ${ }^{1}$ University of Oxford, Department of Physics - Theoretical Physics, 1 Keble Road, Oxford OX1 3NP, U.K. \\ ${ }^{2}$ Theoretische Physik, Fachbereich 8, Bergische Universität Wuppertal, D-42097 Wuppertal, Germany
}

\begin{abstract}
We study a model of directed percolation (DP) with immunization, i.e. with different probabilities for the first infection and subsequent infections. The immunization effect leads to an additional nonMarkovian term in the corresponding field theoretical action. We consider immunization as a small perturbation around the DP fixed point in $d<6$, where the non-Markovian term is relevant. The immunization causes the system to be driven away from the neighbourhood of the DP critical point. In order to investigate the dynamical critical behaviour of the model, we consider the limits of low and high first infection rate, while the second infection rate remains constant at the DP critical value. Scaling arguments are applied to obtain an expression for the survival probability in both limits. The corresponding exponents are written in terms of the critical exponents for ordinary DP and DP with a wall. We find that the survival probability does not obey a power law behaviour, decaying instead as a stretched exponential in the low first infection probability limit and to a constant in the high first infection probability limit. The theoretical predictions are confirmed by optimized numerical simulations in $1+1$ dimensions.
\end{abstract}

PACS numbers: 05.70.Ln, 64.60.Ak, 64.60.Ht

\section{INTRODUCTION}

Epidemic processes can be described as the spread and decay of a non-conserved agent, an example of which is an infectious disease [1]. The agent is not allowed to appear sponstaneously but it can multiply itself by infecting neighbouring individuals, or decay at a constant rate. Depending on the balance between these two processes, the infection may either die out or spread over the entire population. The two regimes of survival and extinction of the epidemic are typically separated by a continuous non-equilibrium phase transition. When the decay process dominates, the epidemic dies out at large times and the system gets trapped in an absorbing state from which it cannot escape.

Continuous phase transitions into absorbing states are associated with certain universality classes 2, 3]. For epidemic processes, a well studied case is the universality class of directed percolation (DP). It is believed that two-state spreading processes with short-range interactions generically belong to the DP class, provided that quenched randomness, unconventional symmetries and large scales due to memory effects are absent [4, 5]. Examples of physical systems whose critical behaviour is described by DP include heterogeneous catalysis [6], chemical reactions [7, 8], interface depinning [9, 10, the onset of spatio-temporal chaos [1], flowing sand 12 and self-organized criticality [13].

The epidemic process in which the susceptibility to infection is independent of previous infections is described by DP. However, for a more realistic description, we

*Electronic address: jimenez@thphys.ox.ac.uk should consider an immunization effect 14]. Immunization can be added to the DP model by changing the susceptibility after the first infection 15, 16, 17]. A minimalistic model that captures this feature is one that is controlled by two independent parameters: a probability of first infection and another probability for all subsequent reinfections. The fact that the local susceptibility depends on whether a site has been infected in the past or not leads to a non-Markovian epidemic process, in which the time evolution depends on the entire history. This non-Markovian feature changes the universality class of the epidemic spreading.

The phase diagram of an epidemic process with immunization (Fig. [1) was studied in Ref. 18, 19]. If the probabilities for first infections and reinfections are equal, the model corresponds to ordinary directed percolation. However if the susceptibility changes to zero after the first infection there is perfect immunization, and the model reduces to the General Epidemic Process (GEP) 1]. GEP belongs to the ordinary percolation universality class 20 . The critical points of the GEP and DP are connected by a curved phase transition line separating a phase in which the spreading process always dies out, from another phase of annular growth, where an active front may propagate into regions of non-immune sites, leaving a bulk of immune sites behind. As shown in Ref. 18] the critical behavior along this line (except for the upper terminal point) belongs to the same universality class as GEP. Using field theoretic renormalization group techniques, the critical exponents were calculated along this line [16, 17]. The main result in Ref. 18] is that the compact growth/no growth phase transition line is at the critical value of the reinfection rate and independent of the first infection rate. Above this horizontal transition line in Fig. 1 the model exhibits compact growth and approaches the stationary state of supercritical DP. This is because, in the active phase of an epidemic process 


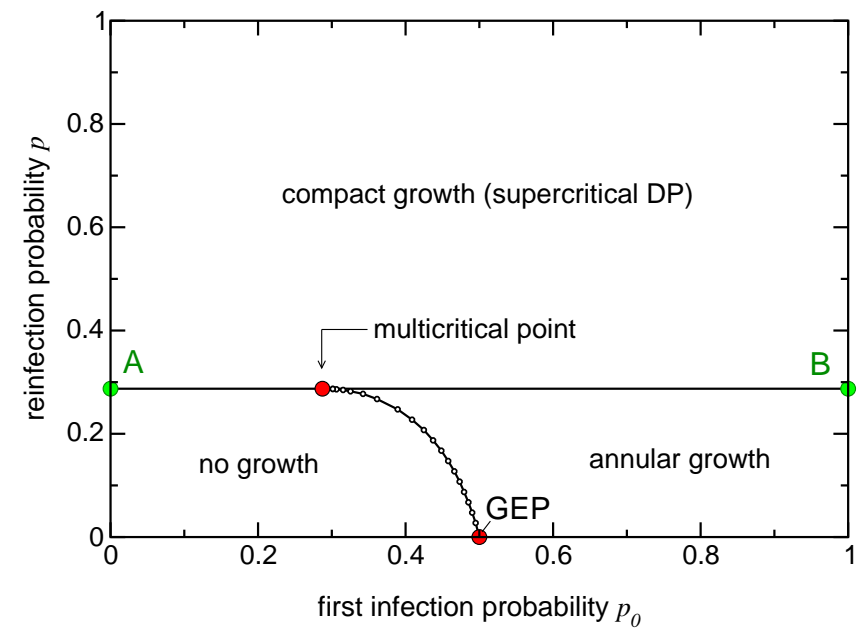

FIG. 1: Phase diagram of directed bond percolation with immunization in $2+1$ dimensions. Along the curve phase transition line the universality class corresponds to the GEP. The horizontal line separates the no growth-annular growth region from the compact growth behaviour. The point where both phase transitions lines meet correspond to the universality class of DP.

with immunization, each site will be visited at least once after a sufficiently long time so that the dynamics in the stationary active state involves only reinfections. On the horizontal phase transition line itself all reinfection processes are critical DP while the probability of first infections may be sub- or supercritical. By varying the first infection rate, we can impede or facilitate the spreading into non-immune regions. In Ref. 18] a numerical analysis of the scaling behaviour along this horizontal line gives no hint of power law behaviour, and it is also suggested that this result could be applied to models with multiple absorbing states 19, 21, 22, 23, 24, 25, 26, 27, 28].

The aim of the present work is to investigate in further detail the dynamical critical behavior along the horizontal phase transition line and in the vicinity of the DP critical point, and to give a theoretical explanation of the absence of power law scaling along this line.

In order to describe the effect of immunization in an epidemic process, in Sec. IIwe study a field theoretic formulation of directed percolation with immunization and we show that the non-Markovian term which contains the immunization effects, is relevant under renormalization group analysis. As a consequence of this result we argue that the asymptotic spreading properties along the horizontal transition line should be determined by the limits of very low and very high first infection probability. Therefore, in Sec. III] we present a study of the very small infection probability limit and we develop a quasi-static approximation to obtain the scaling behaviour of the survival probability of the epidemic. It turns out that this does not follow a power law, but instead decays asymptotically as a stretched exponential. This theoretical pre- diction is confirmed by optimized numerical simulations in $1+1$ dimensions. The high first infection probability limit is studied in Sec. IV giving similar results. We complete the section with a theoretical approximation for the spreading behaviour, and with numerical calculations to corroborate the theoretical claims. Finally, a discussion of these results together with a possible connection with multiple absorbing state models is the subject of the con-

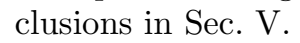

\section{FIELD THEORETICAL ANALYSIS}

\section{A. The model}

In this section we develop an alternative derivation of the action for the DP model with immunization that was proposed in 15, 16, 17]. The microscopic rules for DP in $d+1$ dimensions are rather simple. An infected site at time $t$ can infect its nearest neighbours at time $t+1$ with a probability $p_{0}$. There is a critical threshold $p_{c}$ such that for $p_{0}<p_{c}$ the epidemic process always dies out, that is, it reaches the absorbing state. For $p_{0}>p_{c}$ there is a finite probability that the epidemics survives. At the critical point $p_{0}=p_{c}$ the system scales anisotropically in time and space. The upper critical dimension is $d_{c}=4$, below which the fluctuation effects become important. The field theoretic action of DP 29, 30] reads as follows:

$$
S_{\mathrm{DP}}=\int d t d^{d} x\left[\tilde{\phi}\left(\partial_{t}-D \nabla^{2}+r\right) \phi+u_{1} \tilde{\phi} \phi^{2}-u_{2} \tilde{\phi}^{2} \phi\right] .
$$

Here, $\phi$ is the local activity, $\tilde{\phi}$ is the response field and $r \propto p_{c}-p_{0}$ is the mass parameter which measures the distance from criticality. This action can also be written as a Langevin-type equation for the local activity,

$$
\left(\partial_{t}-D \nabla^{2}+r\right) \phi+\frac{1}{2} u \phi^{2}+\xi(\mathbf{x}, t)=0,
$$

where $u$ is the symmetrized coupling constant after rescaling the fields according to the DP time reversal symmetry. The noise $\xi(\mathbf{x}, t)$ is Gaussian, and satisfies $\langle\xi(\mathbf{x}, t)\rangle=0$ and $\left\langle\xi(\mathbf{x}, t) \xi\left(\mathbf{x}^{\prime}, t^{\prime}\right)\right\rangle=u \delta^{d}\left(\mathbf{x}-\mathbf{x}^{\prime}\right) \delta\left(t-t^{\prime}\right)$. For a systematic analysis of the immunization around the DP fixed point it is often more convenient to make use of the description in terms of the action.

To add immunization to the model, the susceptibility after the first infection is decreased by an amount $\lambda>$ 0 . The probability of infection, $p$, depends locally on position and time, $p=p(\mathbf{x}, t)$. The microscopic rules are modified as follows: a healthy site can first be infected with infection probability $p_{0}$. A site which is infected at time $t$ will become immune at the next time step $t+$ 1. Meanwhile any immune site can be re-infected at the lower re-infection probability $p_{0}-\lambda$. Thus, the state of 
the system at time $t$ depends not just on the configuration of infected sites at time $t-1$, but on the entire previous history.

In order to take into account the effects of immunization, we will modify the DP action. Let us consider a discrete $d+1$ dimensional lattice and assume that the field $\phi(\mathbf{x}, t)$ is almost constant between times $t$ and $t+\Delta t$, where $\Delta t=1$ is the time step unit on the lattice. We then subdivide it into $N$ intervals, $\Delta t / N$. First of all, we want to find an expression for the probability that there is an active site in the temporal sub-interval $\Delta t / N$. To do so in terms of the field $\phi(\mathbf{x}, t)$, we need to take into account the fact that $\phi$ has units of length $h^{-d / 2}$. Then this probability can be written as $w \phi(\mathbf{x}, t) \Delta t / N$, where we introduce a parameter $w$ to ensure that this expression is dimensionless.

In this way, $1-w \phi(\mathbf{x}, t) \frac{\Delta t}{N}$ is the probability that the site is not active in the interval $\Delta t / N$. The probability of not finding activity between times $t$ and $t+\Delta t$, can then be expressed as:

$$
\begin{aligned}
& \prod_{i=0}^{N}\left(1-w \phi(\mathbf{x}, t+i / N) \frac{\Delta t}{N}\right) \sim\left(1-w \phi(\mathbf{x}, t) \frac{\Delta t}{N}\right)^{N} \\
& \stackrel{N \rightarrow \infty}{\longrightarrow} e^{-w \phi(\mathbf{x}, t) \Delta t}
\end{aligned}
$$

However, between times $t=0$ and $t>1$, we can no longer assume that the field $\phi$ is constant. Therefore, the probability of a site $\mathbf{x}$ has never been infected by time $t$ turns out to be,

$$
\prod_{t^{\prime}<t}^{t} e^{-w \phi\left(\mathbf{x}, t^{\prime}\right) \Delta t}=e^{-w \sum_{t^{\prime}=0}^{t} \phi\left(\mathbf{x}, t^{\prime}\right) \Delta t} .
$$

When the continuous limit is taken on the discrete lattice, the probability of having been infected at least once in the past becomes $1-\exp \left(-w \int_{0}^{t} \phi\left(\mathbf{x}, t^{\prime}\right) d t^{\prime}\right)$. This expression is the probability for a site to be immune at time $t$. Since $\lambda$ is the parameter related to the immunization in the system, we can use the above result to write an expression for $p(\mathbf{x}, t)$,

$$
p(\mathbf{x}, t)=p_{0}-\lambda\left[1-e^{-w \int_{0}^{t} \phi\left(\mathbf{x}, t^{\prime}\right) d t^{\prime}}\right] .
$$

We assume that the mass parameter $r$ can be written as $r \propto p_{c}-p(\mathbf{x}, t)$. Thus, using Eq. [5] the addition of immunization can be reflected in the action as a modification in the original DP mass parameter by the following substitution:

$$
r \rightarrow r+\lambda\left[1-e^{-w \int_{0}^{t} \phi\left(\mathbf{x}, t^{\prime}\right) d t^{\prime}}\right]
$$

Finally the action for DP with immunization can be written as a modification of the directed percolation action as follows:

$$
\begin{aligned}
S & =S_{\mathrm{DP}}+ \\
& \lambda \int d t d^{d} x \tilde{\phi}(\mathbf{x}, t) \phi(\mathbf{x}, t)\left[1-e^{-w \int_{0}^{t} d t^{\prime} \phi\left(\mathbf{x}, t^{\prime}\right)}\right] .
\end{aligned}
$$

According to the field theory, $w$ is a finite coupling constant. Then in the numerical calculations on a discrete $d+1$ dimensional lattice, $w$ can be considered as a parameter such that the subsequent infection rate is,

$$
p_{0}+\left(p-p_{0}\right)[1-\exp (-w n(\mathbf{x}, t))] \text {. }
$$

Here, the function $n$ counts all the past activity at a site $\mathbf{x}$ until time $t$. Since the exponential function vanishes at sufficient large times, the effective subsequent infection rate is equal to $p$.

In the numerical calculations carried out in the present work, we assume that the susceptibility for spreading only changes at the first infection process and remains constant thereafter. This assumption is equivalent to take an infinite value of $w$, but does not make any relevant change in the final results of the theory as it is argued in Ref. [18].

\section{B. Renormalization group analysis}

Expanding in Eq. 7 the exponential function as a power series, the action reads

$$
\begin{aligned}
S= & S_{\mathrm{DP}}+\int d t d^{d} x \tilde{\phi}(\mathbf{x}, t) \phi(\mathbf{x}, t) \times \\
& {\left[-\sum_{n=1}^{\infty} \frac{\lambda^{(n)}}{n !}\left(-\int_{0}^{t} d t^{\prime} \phi\left(\mathbf{x}, t^{\prime}\right)\right)^{n}\right], }
\end{aligned}
$$

where we abbreviate $\lambda^{(n)}=\lambda w^{n}$. Let us now study the stability of the DP fixed point with respect to a small perturbation in $\lambda^{(n)}$ by dimensional analysis. Introducing a length scale $k^{-1}$, the mean-field fixed point of DP is characterized by the dimensions $[\tilde{\phi} \phi]=k^{d},[D]=\omega k^{-2}$, and $[u / D]=k^{4-d / 2}$ so that the upper critical dimension is $d_{c}=4$. When immunization is considered as a perturbation, the effective expansion parameter in the expression of the two point vertex function is $u_{2}^{n} \lambda^{(n)}$. Thus, by dimensional analysis we find

$$
\left[\frac{u_{2}^{n} \lambda^{(n)}}{D_{0}^{2 n+1}}\right]=k^{2+n(4-d)} .
$$

Consequently the upper critical dimension for each coupling constant $\lambda^{(n)}$ is $d_{c}=\frac{2}{n}+4$, where $n=1, \ldots, \infty$. This means that for dimensions $d>6, \lambda^{(n)}$ is irrelevant 


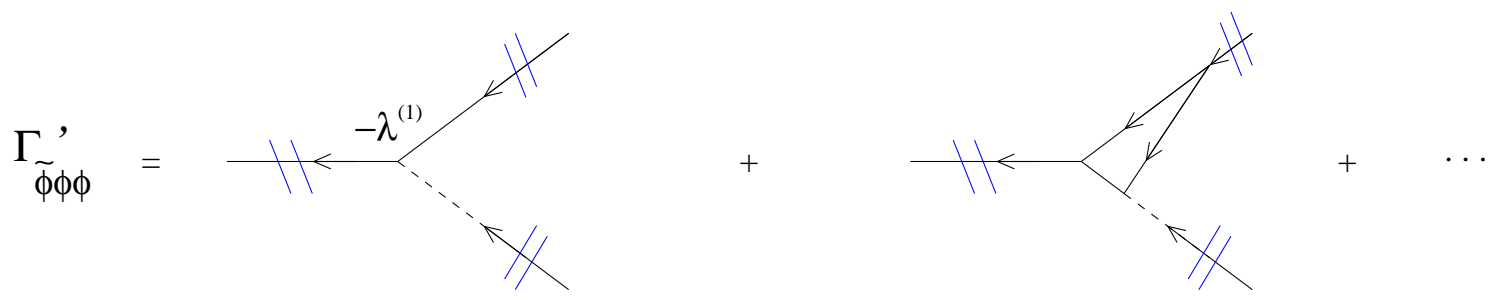

FIG. 2: One-loop diagrams for the three point vertex function $\Gamma_{\tilde{\phi} \phi \phi}^{\prime}$. The dashed lines in the external dashed-continue-leg represent the shift in time indicated by the $\Theta\left(t-t^{\prime}\right)$ function in the action after the expansion of the exponential. The 'external legs' are cut off, and this fact is indicated with the two parallel lines.

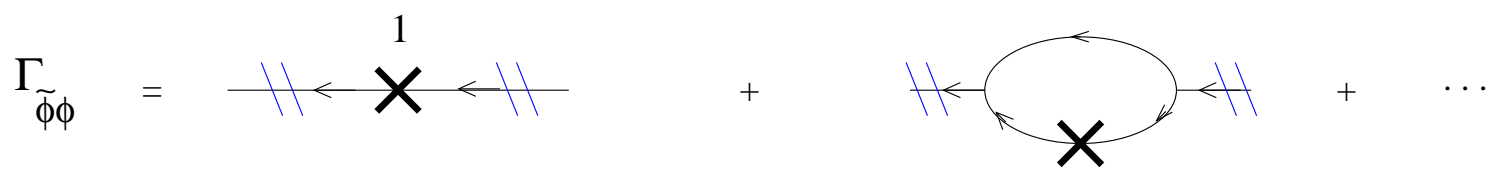

FIG. 3: One-loop diagrams for the two point vertex function $\Gamma_{\tilde{\phi} \phi}$. The 'external legs' are cut off, and this fact is indicated with the two parallel lines. The $\mathbf{X}$ indicates the insertion of the composite operator $\tilde{\phi} \phi$.

for all $n$. The parameter $u_{2}$ will also be irrelevant, and only the mass parameter $r$ renormalizes.

Consider now the renormalization group ( $\mathrm{RG}$ ) parameter space spanned by $r, u_{2}$, and the $\lambda^{(n)}$. The DP fixed point occurs at a point on the $u_{2}$ axis, and the GEP fixed point is somewhere in the hyper-dimensional space. Since all the coupling constants are irrelevant in the case $d>6$, the RG flows bring the system from the neighborhood of the DP fixed point towards the Gaussian DP fixed point, which corresponds to the DP free-field theory. Therefore if close to the DP fixed point we turn on a small perturbation of immunization in a system, the qualitative nature of the system will not change, and the epidemic process is expected to spread according to a mean-field DP process controlled by the probability of first infections.

For $4<d \leq 6$, the coupling constant $\lambda^{(1)}$ is the most relevant. Consequently the higher order terms in the expansion of the exponential in Eq. (9) can be neglected. In this case the RG flows bring the system away from the neighbourhood of the DP fixed point to the GEP fixed point which is stable. Thus, we expect the system to undergo an ordinary crossover from DP to the dynamic percolation universality class at a certain typical time scale. However, recently it has been suggested that dangerous irrelevant operators may possibly lead to a non-trivial critical behavior different from dynamical percolation [28].

To take the fluctuation effects into account, we define the renormalized $\lambda^{(1)}$ as $\lambda_{R}^{(1)}$ and we apply standard methods of the perturbative renormalization group [31]. In particular we make use of an $\epsilon=4-d$ expansion around the DP critical point. The non-Markovian modification in the DP action can be written then as an ad- ditional term of the form,

$$
\lambda^{(1)} \int d t d^{d} x \Theta\left(t-t^{\prime}\right) \tilde{\phi}(\mathbf{x}, t) \phi(\mathbf{x}, t) \phi\left(\mathbf{x}, t^{\prime}\right) .
$$

The non-locality in time, expressed by the $\Theta$ function in Eq. 11], is represented by a dashed line propagator in the Feynman diagrams. The renormalized coupling constant $\lambda_{R}^{(1)}$ can be determined in terms of the vertex function $\Gamma_{\tilde{\phi} \phi \phi}^{\prime}$ (see Fig. 2), evaluated at some specified normalization point, NP, setting a momentum scale $\mu$. The ' in the vertex function's notation in Eq. 11] is used to indicate that this function is calculated in the DP with immunization theory and is different from the one calculated in the ordinary DP. Near the upper critical dimension the ultraviolet divergences are absorbed in the renormalization constant $Z_{\phi}$, where $\phi_{R} \equiv Z_{\phi}^{-1 / 2} \phi$ and $Z_{\phi}=Z_{\tilde{\phi}}$.

$$
\begin{aligned}
\lambda_{R}^{(1)} & =-\left.\Gamma_{R \tilde{\phi} \phi \phi}^{\prime}\right|_{N P} \\
& =-\left.Z_{\phi}^{3 / 2} \Gamma_{\tilde{\phi} \phi \phi}^{\prime}\right|_{N P} .
\end{aligned}
$$

The structure of the corrections to the vertex function $\Gamma_{\tilde{\phi} \phi \phi}^{\prime}$ suggests a correspondence with the RG theory of DP away from the critical point, where is considered the renormalization of a nonzero mass term $r$ which couples with the composite operator $\tilde{\phi}(x, t) \phi(x, t)$. One loop corrections to the corresponding two point bare vertex function $\Gamma_{\tilde{\phi} \phi}$ in this theory, are depicted in Fig. 3. The normalization condition implies that the renormalized two point vertex function $\Gamma_{R \tilde{\phi} \phi}$ evaluated at the normalization point NP, is equal to 1 .

$$
\left.\Gamma_{R \tilde{\phi} \phi}\right|_{N P}=\left.Z_{\tilde{\phi} \phi}^{-1} Z_{\phi} \Gamma_{\tilde{\phi} \phi}\right|_{N P}=1 .
$$


Comparing these corrections with those appearing in the expression to $\Gamma_{\tilde{\phi} \phi \phi}^{\prime}$, it is possible to see that the insertion of the composite operator $\tilde{\phi} \phi$ is equivalent to the "insertion" of the dashed-continuous-line leg in Fig. 2 By inspection of all possible Feynman diagrams, we conclude that this is valid to all orders in perturbation theory. Therefore the Feynman integrals involved in both $\lambda^{(1)}$ and $r$ renormalizations are identical, and we can write:

$$
-\lambda^{(1)} \Gamma_{\tilde{\phi} \phi}=\Gamma_{\tilde{\phi} \phi \phi}^{\prime}
$$

Consequently from Eq. (12), (13) and (14), it can be proved that,

$$
\lambda_{R}^{(1)}=\lambda^{(1)} Z_{\phi}^{1 / 2} Z_{\tilde{\phi} \phi}
$$

To describe how $\lambda_{R}^{(1)}$ flows under renormalization, it is necessary to define a Callan-Symanzik beta function. The dimensionless coupling constant corresponding to $\lambda_{R}^{(1)}$ is

$$
\begin{aligned}
g_{R}^{\prime} & =\left(\frac{\lambda_{R}^{(1)}}{D_{R}^{2}}\right) \mu^{-2-\epsilon / 2} \\
& =\left(\frac{\lambda^{(1)}}{D_{0}^{2}}\right) \mu^{-2-\epsilon / 2} Z_{\phi}^{1 / 2} Z_{\tilde{\phi} \phi} Z_{D}^{-2}
\end{aligned}
$$

where $\epsilon=4-d$, and $D_{R}=D_{0} Z_{D}$ is the renormalized diffusion coefficient. Then the beta function, which gives the differential renormalization group flow equation of $g_{R}$ is

$$
\beta\left(g_{R}^{\prime}\right)=\mu \frac{\partial g_{R}^{\prime}}{\partial \mu}=g_{R}^{\prime}\left[-2-\frac{\epsilon}{2}+\frac{1}{2} \gamma_{\phi}+\gamma_{\tilde{\phi} \phi}-2 \gamma_{D}\right],
$$

where $\gamma_{\phi}, \gamma_{\tilde{\phi} \phi}$ and $\gamma_{D}$ are defined as

$$
\begin{gathered}
\gamma_{\phi}=\mu \frac{\partial \ln Z_{\phi}}{\partial \mu}, \\
\gamma_{\tilde{\phi} \phi}=\mu \frac{\partial \ln Z_{\tilde{\phi} \phi}}{\partial \mu}, \\
\gamma_{D}=\mu \frac{\partial \ln Z_{D}}{\partial \mu} .
\end{gathered}
$$

At the DP fixed point these gamma functions are related to the critical exponents by $\gamma_{\phi}^{*}=\frac{2 \beta}{\nu_{\perp}}-d, \gamma_{\tilde{\phi} \phi}^{*}=z-\frac{1}{\nu_{\perp}}$ and $\gamma_{D}^{*}=z-2$, and thus

$$
\beta\left(g_{R}^{\prime}\right)=\frac{1}{\nu_{\perp}}\left(\beta-\nu_{\|}-1\right) g_{R}^{\prime}+O\left(g_{R}^{\prime 2}\right) .
$$

Then, as we are approaching the infrared limit $(k \rightarrow 0)$, $g_{R}^{\prime}$ increases, being relevant to all orders in perturbation theory.
The renormalization group eigenvalue $y_{\lambda^{(1)}}$, corresponding to $\lambda^{(1)}$, is equal to

$$
y_{\lambda^{(1)}}=\frac{1}{\nu_{\perp}}\left(1+\nu_{\|}-\beta\right) .
$$

Since this expression is always positive, the first term of the expansion in Eq. (9) is relevant to all orders of perturbation theory around $d=d_{c}=4$.

However, for $d<d_{c}=4$ the coupling constants are increasingly relevant in the expansion (9), and it is no longer meaningful to expand the exponential function. The system is driven away from the vicinity of the DP fixed point towards a non-trivial fixed point of order $4-d$. To study this case, we apply a scaling analysis. Assume that position and time scale as $[x]=k^{-1}$ and $[t]=k^{-z}$. In the DP theory away from criticality, the non-zero mass term scales as $[r]=k^{1 / \nu \perp}$. It couples to the operator $\tilde{\phi} \phi$, which has a scaling dimension $x_{\tilde{\phi} \phi}$, such that, $[\tilde{\phi} \phi]=k^{x} \tilde{\phi} \phi$. Using the fact that the action has to be dimensionless, we find that $x_{\tilde{\phi} \phi}=d+z-\frac{1}{\nu_{\perp}}$. Let us focus now on the scaling analysis of the non-Markovian term added to the DP action. First we consider the term corresponding to $n=1$. $\lambda^{(1)}$ scales as $\left[\lambda^{(1)}\right]=k^{y_{\lambda}(1)}$. Notice that the operators $\tilde{\phi}(x, t) \phi(x, t)$ and $\phi\left(x, t^{\prime}\right)$ are evaluated at different space-time points, and consequently they are not correlated. So, each of them scales with its own scaling dimension, $x_{\tilde{\phi} \phi}$ and $x_{\phi}=\beta / \nu_{\perp}$, such that, $[\tilde{\phi}(x, t) \phi(x, t)]=k^{x_{\tilde{\phi} \phi}},\left[\phi\left(x, t^{\prime}\right)\right]=k^{x_{\phi}}$. From the dimensionless nature of the action, we thus obtain $y_{\lambda}=-\frac{1}{\nu_{\perp}}\left(\beta-\nu_{\|}-1\right)$. Generalizing this for higher-order terms in the exponential expansion we find that the scaling exponent for each $\lambda^{(n)}$ are,

$$
y_{\lambda^{(n)}}=\frac{1}{\nu_{\perp}}\left(1+n\left(\nu_{\|}-\beta\right)\right) .
$$

Since $\nu_{\|}-\beta$ is always positive in $d<4$ dimensions, the terms of the expansion (9) are still increasingly relevant.

Furthermore, the scaling invariance of the nonMarkovian term can only be established if the exponential function and its arguments are dimensionless. Therefore the two couplings $w$ and $\lambda$ scale separately with different scaling exponents, i.e., $[\lambda]=k^{y_{\lambda}}$ and $[w]=k^{y_{w}}$. From Eq. (23) we obtain,

$$
y_{\lambda}=\frac{1}{\nu_{\perp}}, \quad y_{w}=\frac{\nu_{\|}-\beta}{\nu_{\perp}} .
$$

Consequently the coupling constant $w$, is also relevant under renormalization.

\section{LOW FIRST INFECTION PROBABILITY LIMIT}

In the previous section using scaling arguments, we showed that the non-Markovian term is relevant for 


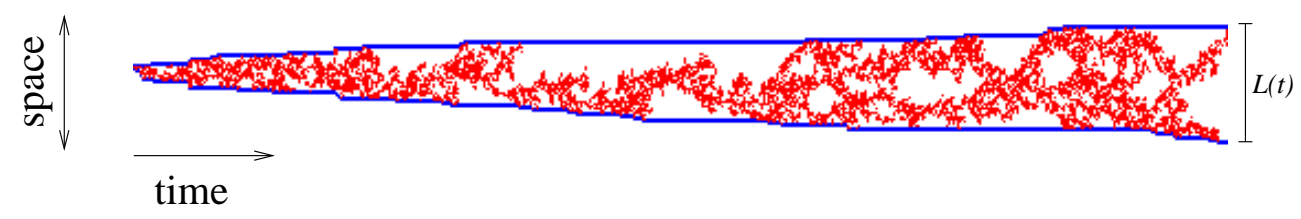

FIG. 4: Spreading in the limit of a very small first infection probability $p_{0}$. The figure shows a surviving cluster in $1+1$ dimensions. In this case the domain of infected/immune is hole-less and grows very slowly with time. Its boundaries can be considered as stationary absorbing walls.

$d<d_{c}=4$. We can now argue that a critical spreading process on the horizontal line in Fig. [1 is therefore driven away from the vicinities of the DP critical point to the points $A$ or $B$, where the probability of first infections is either very low or very high. In order to understand the dynamic behavior in these limits we study the asymptotic spreading properties keeping the second infection probabilities at the critical value of DP. We consider an initial state with a single infected site at the origin in a non-infected environment. For simplicity we will focus on the $1+1$-dimensional lattice. In this case there are not empty sites inside the epidemic clusters, which are composed of infected and immune sites only.

\section{A. Quasi-static approximation}

Let us consider the limit of a very small first infection probability. In this regime, although first infections hardly ever happen, there still exists a surviving critical epidemic process as is shown in Fig. 4 The active domain is composed by re-infected sites among immune ones, and can be considered as bounded by almost rigid "walls" of empty sites. Let us define $L(t)$ to be the distance at time $t$ between these two walls, such that there is at least one site active. If we keep the second infection rate critical, the dynamic epidemic process can be thought as an effective critical DP process evolving in a finite system of size $L(t)$.

A quasi-static approximation is based on the assumption that the time scale on which $L(t)$ grows is much larger than the correlation time of the DP process. Therefore, $L(t)$ can be assumed constant. It is known that in a finite-size system with a constant width $L$, the survival probability of a DP process decays exponentially as $P(t) \sim \exp \left(-t / L^{z}\right)$, where $z=\frac{\nu_{\|}}{\nu_{\perp}}$. Then, in a quasistatic finite system the survival probability can be approximated as $P_{s}(t) \sim \exp \left(-t / \xi_{\|}\right)$, i.e.

$$
\frac{d P_{s}(t)}{d t} \simeq-\frac{1}{\xi_{\|}} P_{s}(t) \simeq-a P_{s}(t) L(t)^{-z},
$$

where $a$ is a non-universal amplitude factor. We need now to know how $L(t)$ grows. Since the two boundaries are absorbing, we can apply the theory of DP with absorbing walls [32]. According to these results, the density of active sites next to the walls generated by the surviving clusters scales as

$$
\rho_{s}(t)=b L(t)^{-\beta_{s} / \nu_{\perp}},
$$

where $b$ is a non-universal amplitude factor which depends on the value of $p_{0} . \quad \beta_{s} \simeq 0.73371(2)$ is the surface critical exponent of DP in $1+1$ dimensions. Clearly, $d L / d t$ is proportional to the frequency by which the system attempts to infect sites at the boundary and thus also to the density of active sites next to the boundary:

$$
\frac{d L(t)}{d t}=p_{0} \rho_{s}(t)=p_{0} b L(t)^{-\beta_{s} / \nu_{\perp}} .
$$

Therefore, the size of the domain grows as

$$
L(t)=\left(p_{0} b\left(1+\beta_{s} / \nu_{\perp}\right) t\right)^{1 /\left(1+\beta_{s} / \nu_{\perp}\right)} .
$$

Inserting this result into Eq. (25) and solving the differential equation we obtain

$$
\ln P_{s}(t) \sim-\int_{0}^{t} L(z)^{-z} d t \sim-p_{0}^{-\alpha} t^{1-\alpha},
$$

where $\alpha=\frac{\nu_{\|}}{\nu_{\perp}+\beta_{s}} \simeq 0.947167$. Hence the survival decays asymptotically as a stretched exponential of the form

$$
P_{s}(t)=P_{s}(0) \exp \left(-A p_{0}^{-\alpha} t^{1-\alpha}\right) .
$$

with $A=\frac{a}{1-\alpha}\left(\frac{\alpha}{b z}\right)^{\alpha}$. The above result implies that the average survival time $T$ is finite and scales as

$$
T \sim p_{0}^{\alpha /(1-\alpha)} .
$$

It is interesting to compare these results with recent findings for random walkers between movable reflectors, where the survival probability was shown to decay as a power law with continuously varying exponents [33]. We note that this result is not in contradiction with the present work since it would correspond to the limit $\alpha \rightarrow 1$.

\section{B. Numerical simulations}

The lattice model considered is a $1+1$ dimensional triangular lattice. We simulate a directed bond percolation 


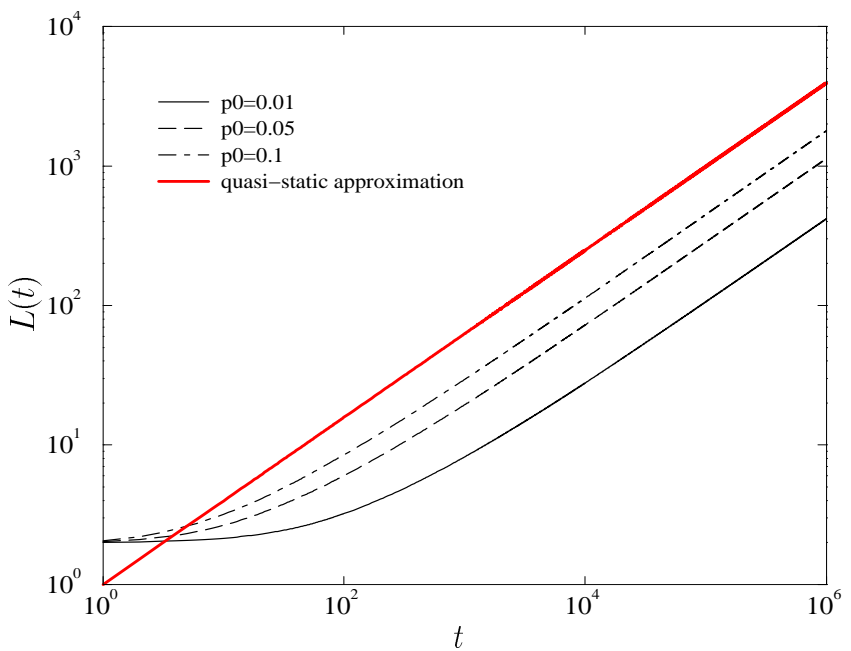

FIG. 5: The logarithm of the domain size $L(t)$ in the problem of directed percolation with immunization for small values of $p_{0}$ as function of time $t$. The numerical results are compared with the quasic-static approximation predictions.

process with a first infection rate $p_{0}$ different from a rate for subsequent infections or second infection rate $p$.

Let us first consider the limit of a very small first infection probability, where it is difficult for the process to infect sites at the boundary that have never been infected before (see Fig. 4). Bonds inside the infected region are open with the critical probability $p=p_{c}=$ 0.6447001(1) [34], while bonds leading to healthy sites at the boundary are open with a different probability of first infection $p_{0}$. However, as $p_{0}$ is very small, conventional seed simulations are not suitable since most of the runs terminate after a very short time. For example, for $p=0.1$ most of the runs survive for less than 100 time steps. Consequently we apply enrichment methods in order to circumvent this limitation. First of all, we apply a very simple enrichment method, in which we consider one lattice system. We keep the activity artificially alive at any time $t$. This is achieved by ignoring the updates which lead to the inactive state. Averaging over many realizations we measured the domain size $L(t)$ and the surface density of active sites. We find that the exponents predicted by the quasi-static approximation are in good agreement with the values obtained with the numerical simulations as shown in Fig. [5] and Fig. [6] The nonuniversal factor $b$ in Eq. 28) depends on the value of $p_{0}$. For, $p_{0}=0.01$ it takes the value $b=1.65$.

To determine the survival probability $P_{s}(t)$, we apply another enrichment method [35], which leads to a considerable improvement. The simulation starts with an ensemble of $N=65536$ independent systems. Whenever the number of active runs becomes smaller than $N / 2$ the ensemble is duplicated by creating identical copies of all the remaining active states. The new systems are labelled according to their ancestors. The survival probability $P_{s}$

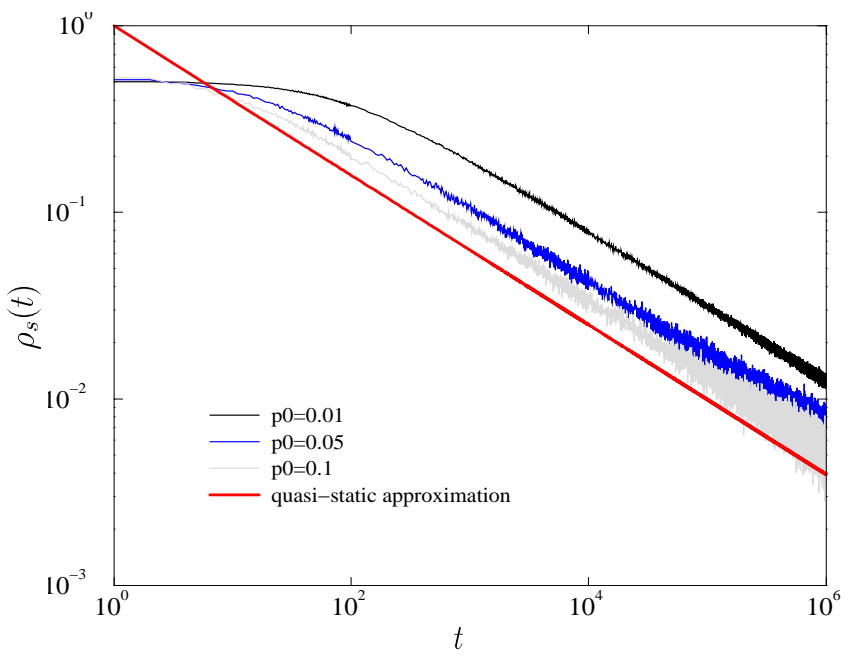

FIG. 6: The logarithm of the surface density of active sites of directed percolation with immunization for small values of $p_{0}$ as function of time $t$. The numerical results are compared with the quasic-static approximation predictions.

then is reduced by a factor of $n_{f} / n_{i}$, where $n_{i}$ is the number of initial active systems and $n_{f}$ is the number of remaining active systems before the duplication. This process may be repeated as long as the ensemble has a sufficiently large number $m$ of independent ancestors at $t=0$. Using this method we were able to extend the temporal range of the simulation by four orders of magnitude up to $t=10^{6}$. Our results are shown in Fig. 7 for various values of $p_{0}$. As can be seen, the survival probability plotted in a double logarithmic representation is not a straight line, proving that it does not follow a power law. For the case $p_{0}=0.01$, the parameter $a$ in Eq. (25) takes the value $a=1.00(2)$.

\section{Lattice effects}

The scaling arguments in Section $11 \mathrm{~A}$ are developed assuming the existence of a well defined mean value for the domain size $L(t)$. To justify this assumption, we define $P(L, t)$ to be the probability of having a domain of size $L$ at time $t$. Then the survival probability is $P_{s}(t)=\int P(L, t) d L$. In this subsection we show that $L(t)$ is peaked around the mean value $L(t)$.

We begin by writing down a heuristic discrete master equation for the temporal evolution of $P(L, t)$ :

$$
\begin{aligned}
P(L, t+1)= & e^{-a L^{-z}} P(L, t)+b p_{0}(L-1)^{-\beta_{s} / \nu_{\perp}} \times \\
& P(L-1, t)-b p_{0} L^{-\beta_{s} / \nu_{\perp}} P(L, t) .
\end{aligned}
$$

The first term in the right hand side of Eq. (32) describes the change in $P(L, t)$ due to terminating runs according to Eq. (25) integrated over the time interval $[t, t+1]$. The second term corresponds to the probability of creating 


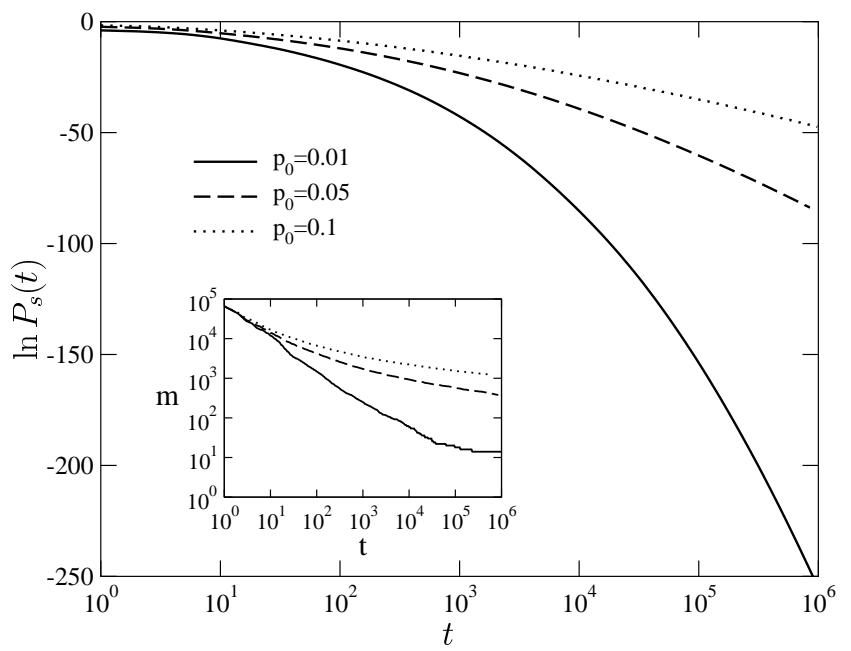

FIG. 7: The logarithm of the survival probability $P_{s}(t)$ of directed percolation with immunization for small values of $p_{0}$. The inset shows the corresponding number of independent ancestors as a function of time (see text). For $p_{0}=0.01$ the duplication method reaches its limit since only 11 independent ancestors are left.

a domain of size $L$ from a domain of length $(L-1)$. The third term describes a loss term corresponding to $L \rightarrow L+1$. We neglect the second order contributions $+b^{2} p_{0}^{2}(L-1)^{-2 \beta_{s} / \nu_{\perp}} P(L-2, t)$ and $-b^{2} p_{0}^{2}(L-1)^{-2 \beta_{s} / \nu_{\perp}}$ which only affect the behaviour at small time and small $L$ regime.

It is straightforward to show that this master equation is consistent with Eq. (25), by summing over all $L$ and replacing $L$ by its average value $L(t)$. On doing this we obtain $d P_{s}(t) / d t=-a L(t)^{-z} P_{s}(t)$, that is the same equation as Eq. (25).

Consider Eq. (32) with $p_{0}, a$ and $b$ fixed at certain values. Then the differential equation which corresponds to the master equation, Eq. (32), can be written as

$$
\begin{aligned}
\frac{\partial P(L, t)}{\partial t}= & -\frac{a}{L^{z}} P(L, t)- \\
& p_{0} b \frac{\partial}{\partial L}\left[L^{-\beta_{s} / \nu_{\perp}} P(L, t)\right], L \gg 1 .
\end{aligned}
$$

The solution for Eq. (33) is

$$
\begin{aligned}
P(L, t)= & e^{-\frac{a}{p_{0} b\left(1-z+\beta_{s} / \nu_{\perp}\right)} L^{1-z+\beta_{s} / \nu_{\perp}}} L^{\beta_{s} / \nu_{\perp}} \\
& \psi\left[L^{\beta_{s} / \nu_{\perp}+1}-p_{0} b\left(\beta_{s} / \nu_{\perp}+1\right) t\right],
\end{aligned}
$$

where $L \gg 1$. Consider the integrated distribution probability $G(L, t)=\int_{L}^{\infty} P(L, t) d L$. Then $G(L, t)$ has the functional form

$$
\begin{aligned}
G(L, t)= & e^{-c\left[p_{0} b\left(\beta_{s} / \nu_{\perp}+1\right) t\right]^{\kappa}} \times \\
& \phi\left[L^{\frac{\beta_{s}}{\nu_{\perp}}+1}-p_{0} b\left(\frac{\beta_{s}}{\nu_{\perp}}+1\right) t\right]\left(1+O\left(\frac{1}{t^{1-\kappa}}\right)\right),
\end{aligned}
$$

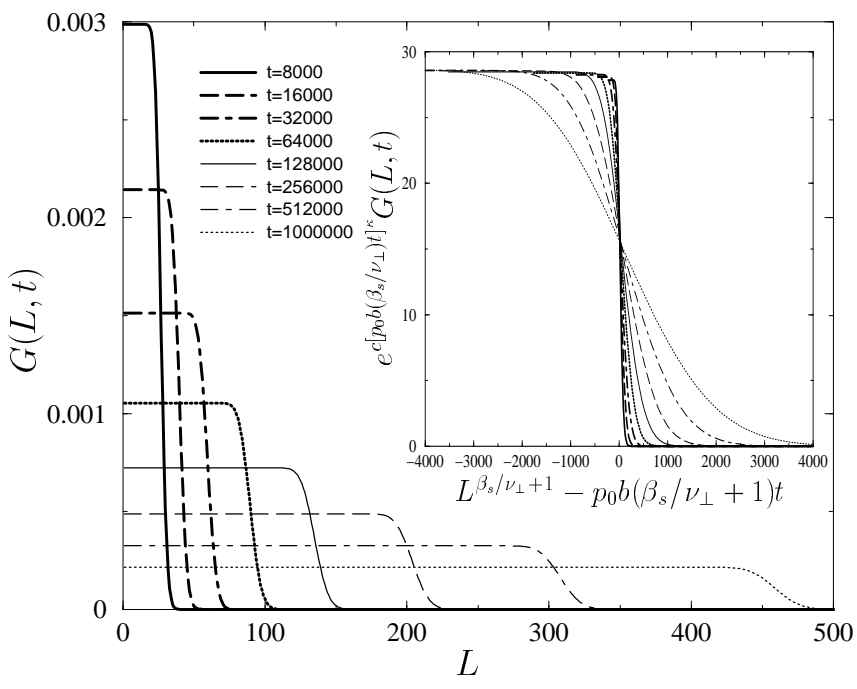

FIG. 8: Probability $G(L, t)=\int_{L}^{\infty} P(L, t) d L$ computed by numerical integration of the master equation Eq. (32). The plots correspond to $a=0.01$ and $p_{0}=0.01$. The inset shows the scaling plot when $G(L, t)$ scales according to Eq. (35).

with $c=a /\left(p_{0} b\left(1-z+\beta_{s} / \nu_{\perp}\right)\right)$ and $\kappa=(1-z+$ $\left.\beta_{s} / \nu_{\perp}\right) /\left(1+\beta_{s} / \nu_{\perp}\right)$.

To observe the qualitative behaviour of $P(L, t)$ and $G(L, t)$, we carry out numerical integrations of Eq. (32). As initial conditions we choose the state in which there is only one site infected at time $t=0$. In Fig. $8 G(L, t)$ is shown for several values of time $t$. It behaves like a propagating front with an overall exponential factor of time $t$. The fact that all the $G(L, t)$ curves intersect at the same point (see inset in Fig. [8), implies that there is a well defined peak and a mean value $L(t)$ for $P(L, t)$. The qualitative behaviour of $G(L, t)$ is independent of the particular values of $a$ and $p_{0}$. Consequently, to perform these calculations, we use values of $a$ and $p_{0}$ such that the effect of the exponential decay in Eq. (35) is not so pronounced.

We now compare the predictions of the master equation proposed in this subsection, with the results obtained from a simulation of the model. In Eq. (32), we fixed the value of $p_{0}=0.01$ and vary $a$. The survival probability calculated from direct numerical integration of Eq. (32), describes well the corresponding simulation result for $p=0.01$, in the case $a=1$ (see Fig. 9).

\section{HIGH FIRST INFECTION PROBABILITY LIMIT}

Let us finally consider the limit of a very high first infection probability $p_{0} \rightarrow 1$. Again we restrict to the 1+1-dimensional case, where the region of immune sites does not contain healthy sites inside. Because of the enhanced spreading probability at the boundaries, the do- 


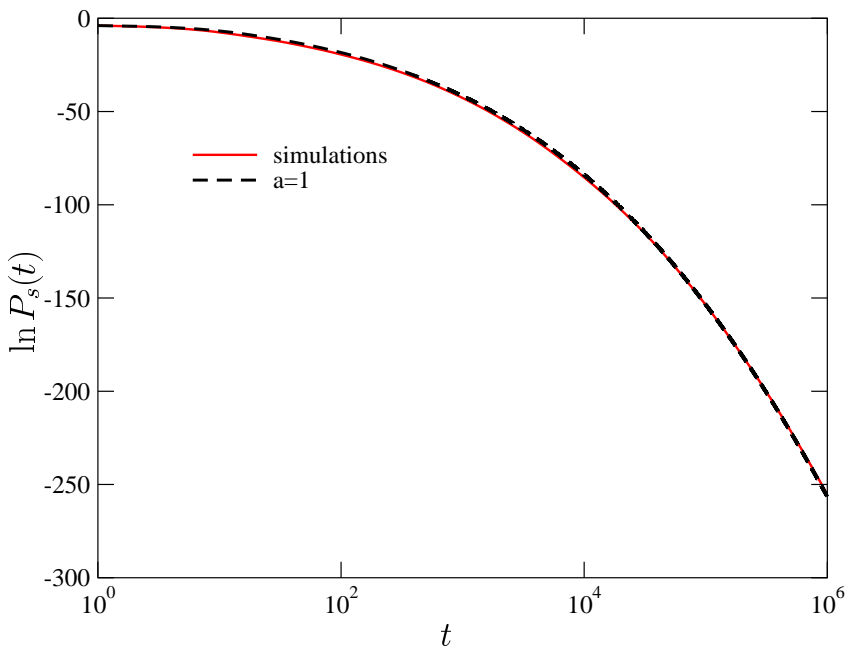

FIG. 9: Survival probability calculated using the master equation Eq. (32). We set $p_{0}=0.01, a=1$ and $b=1.65$. The result is in good agreement with the Monte Carlo simulations for $p_{0}=0.01$.

main grows rapidly by sudden avalanches of successive first infections (see Fig. 10). Since the avalanches at the left and the right boundary are expected to be uncorrelated, it suffices to study the propagation of one of the boundaries. As in the previous section, we propose simple scaling arguments in order to describe the growth of the infected/immune domain and the survival probability.

\section{A. Independent avalanche approximation}

In 1+1-dimensional directed bond percolation with $p=$ $p_{c}$ and $p_{0} \rightarrow 1$ an avalanche is caused by a sequence of open bonds at the boundary. Therefore, the avalanche size $\xi$ is distributed exponentially as

$$
P(\xi)=\left(1-p_{0}\right) p_{0}^{\xi} \sim e^{-\xi / \bar{\xi}},
$$

The quantity

$$
\bar{\xi}=-\frac{1}{\ln p_{0}} \approx \frac{1}{1-p_{0}} \quad p_{0} \rightarrow 1 .
$$

is the average distance by which the avalanche advances the boundary in space. After each avalanche the process continues to evolve as an ordinary critical DP process inside the immune domain until it terminates or returns to the boundary where it releases a new avalanche. Thus the spreading behavior is mainly determined by the distribution of waiting times $\tau$ between the avalanches. We argue that the distribution of waiting times between avalanches is related to the problem of local persistence in DP 36, 37] (for a recent review on persistence see e.g. 38]). The local persistence probability $R(t)$ is defined as the probability that a randomly selected site in

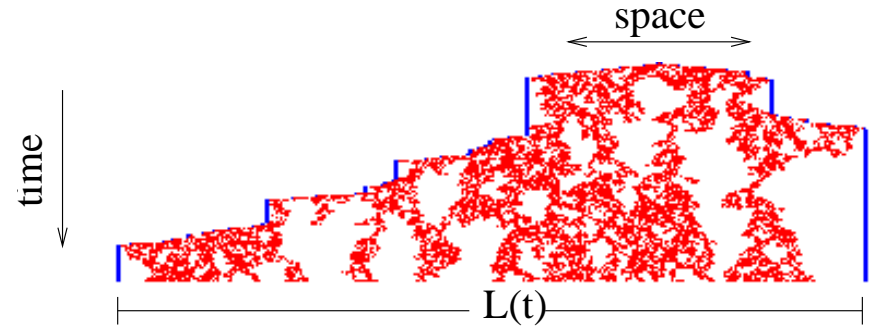

FIG. 10: Spreading in the limit of a very high first infection rate.

an ordinary critical DP process starting from a homogeneous initial state has not been reactivated until time $t$. It was shown that this quantity decays algebraically as $R(t) \sim t^{-\Theta}$, where $\Theta=1.50(1)$ is the so-called local persistence exponent 36 . In the present problem the situation is similar: Each avalanche creates locally a quasi-homogeneous state. The process then evolves as an ordinary critical DP process inside the infected/immune region until the boundary is revisited for the first time in order to release a new avalanche. However, unlike persistence studies in $1+1$ dimensions, where a persistent site can be activated independently from the left and from the right, the boundary sites in the present problem can be infected only from one side. Hence the probability that the next avalanche has not yet been released decays as $\tau^{-\Theta / 2}$. Thus we conjecture that the waiting times between avalanches are distributed algebraically as

$$
P(\tau) \sim \tau^{-1-\Theta / 2} .
$$

Next, we argue that these waiting times may be interpreted as directed Lévy flights in time [39]. After each flight the domain size $L(t)$ grows on average by the mean avalanche size $\bar{\xi}$. This type of growth may be described by the equation

$$
D_{t}^{\Theta / 2} L(t) \sim \bar{\xi}
$$

where $D_{t}^{\Theta / 2}$ is a fractional derivative defined through its action in Fourier space $D_{t}^{\Theta / 2} e^{i \omega t}=(i \omega)^{\Theta / 2} e^{i \omega t}$. Simple dimensional analysis leads to the result

$$
L(t) \sim \bar{\xi} t^{\Theta / 2} .
$$

In order to compute the survival probability, we note that during the waiting time the outermost active site departs from the boundary with an average distance $\ell(\tau) \sim \tau^{1 / z}$. Obviously, the process can only terminate if this distance is of the same order as the size of the infected/immune domain $L(t)$. Therefore, we expect the distribution of waiting times between avalanches to be cut off by a maximal waiting time

$$
\tau_{\max } \sim L^{z}(t) \sim \bar{\xi}^{z} t^{z \Theta / 2} .
$$

Consequently, the probability that the process terminates between two avalanches is given by

$$
P_{0}=\frac{\int_{\tau_{\max }}^{\infty} d \tau P(\tau)}{\int_{1}^{\infty} d \tau P(\tau)} \sim \tau_{\max }^{-\Theta / 2}
$$


On the other hand, the cutoff due to terminating runs implies that the average waiting time is finite and scales as

$$
\bar{\tau}=\frac{\int_{1}^{\tau_{\max }} d \tau \tau P(\tau)}{\int_{1}^{\tau_{\max }} d \tau P(\tau)}=\tau_{\max }^{1-\Theta / 2}
$$

Therefore, the average loss of the survival probability per unit time is given by $P_{0} / \bar{\tau} \sim 1 / \tau_{\max }$, i.e.

$$
\frac{d P_{s}(t)}{d t} \sim-P_{s}(t) / \tau_{\max } \sim-P_{s}(t) \bar{\xi}^{-z} t^{-z \Theta / 2} .
$$

Solving this equation, the asymptotic behavior of the survival probability is not a stretched exponential and is given by a

$$
P_{s}(t)=\frac{1}{\tilde{N}} \exp \left(+\tilde{A} \bar{\xi}^{-z} t^{1-z \Theta / 2}\right),
$$

where $\tilde{N}$ and $\tilde{A}$ are unknown constants. In contrast to the previous case in Eq. (30) the exponent $1-z \Theta / 2 \simeq$ -0.185 is negative. Consequently the survival probability tends to a constant $P_{s}(\infty)>0$, meaning that a finite fraction of runs survives for infinitely long time. For very large $p_{0}$ this constant is expected to scale as

$$
1-P_{s}(\infty) \propto\left(1-p_{0}\right)^{z \Theta / 2} .
$$

\section{B. Numerical results}

In order to verify these results numerically, we developed an especially optimized Monte Carlo algorithm for large values of $p_{0}$. In order to compute the survival time for a run and to see how the boundaries advance for a given realization of open and closed bonds, it is in most cases not necessary to construct the entire cluster. Rather it suffices to construct its branches next to the boundaries. For example, in Fig. 10] large parts of the cluster are irrelevant for the advancement of the boundaries and the survival time. For this reason our algorithm first constructs the branches of the cluster next to the boundaries. If these branches terminate before the simulation time is reached, we recursively constructs the omitted branches in the interior of the clusters. If however they do not terminate, then the recursive construction is not performed. Using this technique we could extend the simulation time by two decades to $10^{4}$ time steps, two decades less than in the previous case of low $p_{0}$.

Our numerical results are shown in Fig. 11] The left panel shows the survival probability as a function of time in a directed bond percolation process with $p_{0}=$ $0.85,0.90,0.95$, and 0.98 . The positive curvature of the lines indicates that there is no power-law scaling. Although the upward curvature is in agreement with the expected result (45), the simulation time is not large enough to confirm the behaviour of Eq. (45) quantitatively, mainly because the constant $\tilde{A}$ is not known. In order to substantiate the assumptions made in the previous subsection, we first measured the growing domain size $L(t)$. As shown in Fig. 11], the measured slope seems to tend to the predicted slope $\Theta / 2=0.75(1)$. Moreover, we estimated the logarithmic derivative of the survival probability, in which the unknown prefactor $\tilde{A}$ drops out (see right panel of Fig. 11). Although this data set is quite noisy, we observe a rather clean power law. The estimated slope 1.12(10) is consistent with the theoretical prediction $t^{-z \Theta / 2}=t^{-1.185}$, supporting the assumptions which led to the result (45).

\section{CONCLUSIONS}

We analyzed the effects of immunization as a small perturbation on the DP model and studied in detail the scaling behaviour of the theory around the DP critical point. We derived by an alternative method the field theoretic action for the model. A non-Markovian term is added to the DP action because of the presence of immunization. In the field theory, the probability for subsequent infections, which is different from the first infection probability, is a function of position and time. Nevertheless, we assumed in the lattice model that the susceptibility for spreading changes only after the first infection, remaining constant thereafter. This assumption does not changes the final results.

The phase diagram (cf. Fig. 11) comprises two phase transition lines, namely, a horizontal line, where the process in reinfected regions shows the critical behavior of DP, and (in more than one spatial dimension) a curved transition line, where the critical behavior corresponds to the general epidemic process studied in [18]. Both lines meet at the DP critical point $p=p_{0}=p_{c}$.

The non-Markovian term turns out to be relevant for $d<6$. We considered the RG flows and argued that any point in the neighbourhood of the DP critical point will be driven away from it. In particular, we focused our study on the horizontal phase transition line with a critical reinfection probability $p=p_{c}$. The system along this line, is driven away from the DP critical point as soon as the immunization effects are turned on as a small perturbation. The asymptotic behavior is determined by the limits of very low and high first infection probability (close to points $A$ and $B$ in Fig. (1).

We proposed simple scaling arguments for the behaviour of the survival probability in both limits. We related the corresponding exponents with those exponents of the critical ordinary DP theory and DP near a wall. The survival probability is found to obey a stretched exponential behaviour in the low first infection probability limit, and it decays to a constant in the high first infec- 


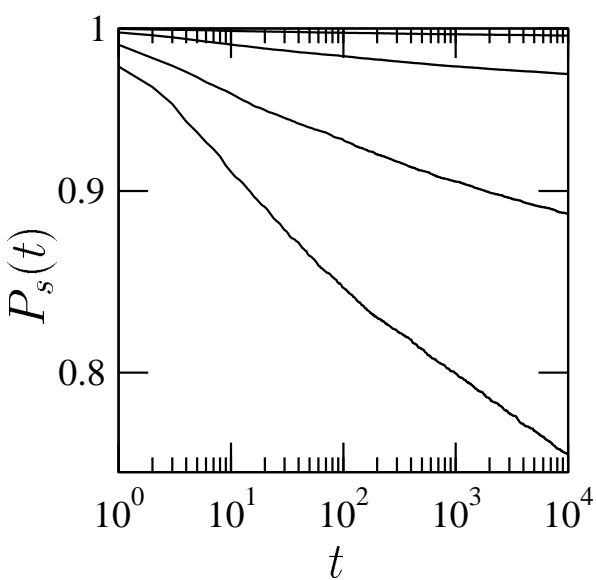

(a)

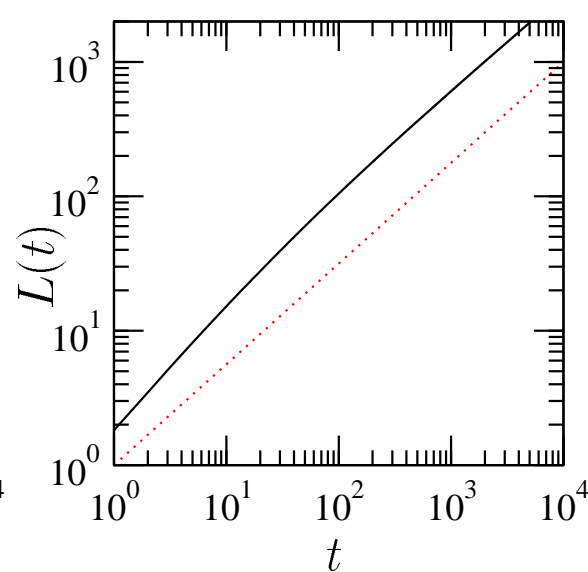

(b)

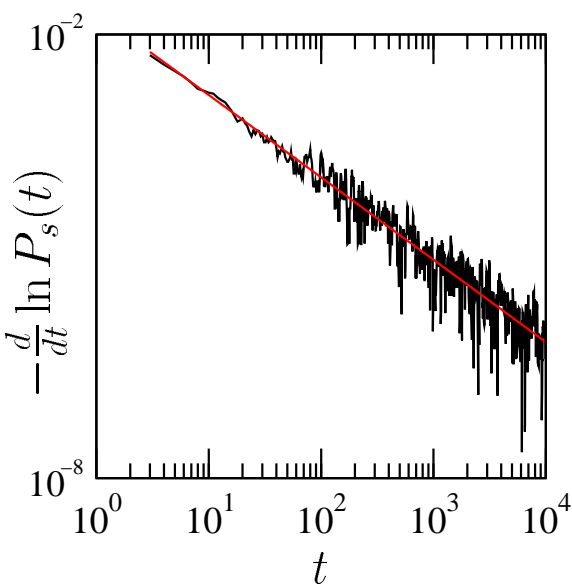

(c)

FIG. 11: Avalanche approximation in comparison with numerical results. (a) Numerically determined survival probability for $p_{0}=0.85,0.9,0.95$, and 0.98 from bottom to top. (b) Numerically determined domain size $L(t)$ for $p_{0}=0.9$ (solid line) compared to the theoretical prediction (40) (dotted line). (c) Logarithmic derivative of the survival probability for $p_{0}=0.9$.

tion probability limit, taking the form

$$
P_{s}(t) \propto \begin{cases}\exp \left(-a t^{+0.0528}\right) & \text { for } p_{0} \rightarrow 0 \\ \exp \left(+b t^{-0.185}\right) & \text { for } p_{0} \rightarrow 1\end{cases}
$$

The numerical simulations in $1+1$ dimensions support these theoretical predictions, ruling out the possibility of asymptotic power-law scaling in both limits. The question as to whether the stretched exponential decay of the survival probability persists in higher dimensions is still open.

Finally, we comment on a possible connection of these results with the problem of infinitely many absorbing states. A typical example of such systems is the pair contact process $2 A \rightarrow 3 A, 2 A \rightarrow \emptyset$, in which solitary particles are not allowed to diffuse [40]. In Ref. [19, 24, 25, 26] this model was studied with an effective Langevin equation and it was inferred that the survival probability decays as a power law at the transition point, with continuously varying exponents. However, the field theoretical action studied in [19, 24, 26] is identical to the one studied in this paper. Hence, we conclude that, according to the analysis presented in this paper, seed simulations of the pair contact process, at least in $1+1$ dimensions, should show an stretched exponential behaviour and not a power low decay at the transition point.

\section{Acknowledgments}

We want to thank Peter Grassberger for communication of unpublished numerical calculations. A.J.D. is greatly indebted to John Cardy for enlightening discussions and suggestions, and for his most careful reading of the manuscript. A.J.D. would also like to thank to R. Rajesh for useful comments.

Part of the present computations have been carried out on the 128-node Alpha Linux Cluster Engine ALiCE at Wuppertal University. We thank N. Eicker, Th. Lippert and B. Orth for their assistance.

A.J.D. was supported by CONICET (Argentina), the British Council-Fundación Antorchas (Argentina), and the ORS Award Scheme (UK).
[1] D. Mollison, J. R. Stat. Soc. B 39, 283 (1977).

[2] J. Marro and R. Dickman, Nonequilibrium phase transitions in lattice models, Cambridge University Press, Cambridge, 1999.

[3] H. Hinrichsen, Adv. Phys. 49, 815 (2000).

[4] H. K. Janssen, Z. Phys. B 42, 151 (1981).

[5] P. Grassberger, Z. Phys. B 47, 365 (1982).

[6] R. M. Ziff, E. Gulari and Y. Barshad, Phys. Rev. Lett 56, 2553 (1986).
[7] P. Grassberger and A. De la Torre, Ann. Phys. 122, 373 (1979).

[8] F. Schlögl, Z. Phys. 253, 147 (1972).

[9] L. H. Tang and H. Leschhorn, Phys. Rev. A 45, R8309 (1992).

[10] S. V. Buldyrev, A.-L. Barabási, F. Caserta, S. Havlin, H. E. Stanley and T. Vicsek, Phys. Rev. A 45, R8313(1992).

[11] P. Rupp, R. Richter, and I. Rehberg, Phys. Rev. E 67, 036209 (2003). 
[12] H. Hinrichsen, A. Jiménez-Dalmaroni, Y. Rozov, and E. Domany, Phys. Rev. Lett. 83, 4999 (1999);

J. Stat. Phys. 98, 1149 (2000).

[13] P. K. Mohanty and Deepak Dhar, Phys. Rev. Lett. 89, 104303 (2002).

[14] A. S. Perelson and G. Weisbuch, Rev. Mod. Phys. 69, 1219 (1997).

[15] J. L. Cardy, J. Phys. A: Math. Gen. 16, L709 (1983).

[16] J. L. Cardy and P. Grassberger, J. Phys. A 18, L267 (1985).

[17] H. K. Janssen, Z. Phys. B 58, 311 (1985).

[18] P. Grassberger, H. Chaté and G. Rousseau, Phys. Rev. E 55, 2488 (1997).

[19] M. A. Muñoz, G. Grinstein and R. Dickman, J. Stat. Phys. 91, 541 (1998).

[20] Z. Alexandrowicz, Phys.Lett. 80A,284 (1980).

[21] I. Jensen and R. Dickman, Phys. Rev. E 48, 1710 (1993).

[22] J. F. F. Mendes, R. Dickman, M. Henkel, and M. C. Marques, J. Phys. A 27, 3019 (1994).

[23] R. Dickman, Phys. Rev. E 53, 2223 (1996).

[24] M. A. Muñoz, G. Grinstein, R. Dickman, and R. Livi, Phys. Rev. Lett. 76, 451 (1996).

[25] C. Lopez and M. Muñoz, Phys. Rev. E 56, 4864 (1997).

[26] M. A. Muñoz, G. Grinstein, R. Dickman, and R. Livi, Physica D 103, 485 (1997).
[27] W. Hwang and H. Park, Phys. Rev. E 59, 4683 (1999).

[28] F. van Wijland, Phys. Rev. Lett. 89,190602 (2002).

[29] P. Grassberger and K. Sundermeyer, Phys. Lett. B 77, 220 (1978).

[30] J. L. Cardy and R. L. Sugar, J. Phys. A 13, L432 (1980).

[31] D. Amit, "Field Theory, the Renormalization Group, and Critical Phenomena", World Scientific (1984).

[32] P. Fröjdh, M. Howard, and K. B. Lauritsen, Int. J. Mod. Phys. 15, 1761 (2001), and references therein.

[33] R. Dickman and D. ben-Avraham, Phys. Rev. E 64, 020102R; R. Dickman and D. ben-Avraham, eprint cond-mat/0304292

[34] I. Jensen, Phys. Rev. Lett. 77, 4988 (1996).

[35] P. Grassberger, Comput. Phys. Commun 147 (1-2), 64 (2002).

[36] H. Hinrichsen and H. M. Koduvely, Eur. Phys. J. B 5, 257 (1998).

[37] K. Oerding and F. van Wijland, J. Phys. A 31, 7011 (1998).

[38] For a review see S. N. Majumdar, Current Science 77, 370 (1999).

[39] J.-P. Bouchaud and A. Georges, Phys. Rep. 195, 127 (1990).

[40] I. Jensen, Phys. Rev. Lett. 70, 1465 (1993). 